\title{
The role of echocardiography in the assessment of hypertrophic cardiomyopathy
}

\author{
Harry Rakowski, John Fulop and E. Douglas Wigle
}

Division of Cardiology, Department of Medicine, Toronto General Hospital and University of Toronto, Toronto, Ontario, Canada.

\begin{abstract}
Summary: Echocardiography has greatly simplified the diagnosis of hypertrophic cardiomyopathy and routine haemodynamic studies are usually only required in patients being considered for myectomy or the assessment of coexistent coronary disease. A complete echo Doppler study should be performed in all patients with hypertrophic cardiomyopathy to define the degree of obstruction, the degree of asymmetric hypertrophy and abnormalities of diastolic function. In this manner the patient can be classified according to haemodynamic subgroup, thus influencing the choice of treatment and helping to determine prognosis. These studies also provide a simple quantitative method of assessing the beneficial effects of medical or surgical therapy.
\end{abstract}

\section{Introduction}

In the past 15 years echocardiography has become essential in the diagnosis of hypertrophic cardiomyopathy (HCM) and to understanding its pathophysiology. Initially, antemortem diagnosis required haemodynamic studies, demonstrating dynamic obstruction across the left ventricular outflow tract (LVOT). Echocardiography allowed non-invasive detection and quantitation of the degree of LVOT obstruction and showed it to be due to systolic narrowing of the LVOT by the mitral apparatus. Also the degree and extent of asymmetrical hypertrophy could be defined in a manner previously possible only during pathological studies. This subsequently led to the recognition of non-obstructive forms of the disease as well as the detection of asymptomatic patients. More recently pulsed and continuous wave $(\mathrm{CW})$ Doppler studies have made it easier to assess abnormal systolic flow patterns and to quantitate the degree of LVOT obstruction, associated mitral regurgitation and diastolic dysfunction. As medical and surgical therapy have evolved and improved, echocardiographic and Doppler studies have played an invaluable role in assessing the benefits of therapy.

Establishing the diagnosis and quantitating the degree of LVOT obstruction

The echocardiographic diagnosis of HCM was originally based on two abnormalities, namely, asym-

Correspondence: Harry Rakowski M.D., F.R.C.P.C. metrical septal hypertrophy and systolic anterior motion (SAM) of the mitral apparatus (Popp et al., 1969; Shah et al., 1969). Both abnormalities were initially thought to be specific for HCM but were subsequently shown to occur occasionally in other conditions. This unfortunately led some authors to the incorrect conclusion that they were not useful findings. Better definition of the septum by 2D-echocardiography (Martin et al., 1979) and the use of a septal to posterior wall ratio of greater than 1.5:1 to define asymmetric hypertrophy has helped to increase the specificity of this finding. Similarly, while minor degrees of SAM can occur in other conditions, severe SAM (Figures 1 \& 2) is pathognomonic for dynamic LVOT obstruction. Only occasionally is this seen in the absence of asymmetric hypertrophy and almost always in conditions where there is hyperdynamic LV wall motion and a narrowed LVOT.

In patients with HCM it is important to define the haemodynamic subgroup (resting, latent or no obstruction) since it has major implications for prognosis and treatment. Gilbert et al. (1980) demonstrated the ability to do this non-invasively in an M-mode study of 74 patients with HCM classified haemodynamically. The degree of SAM was defined as severe, moderate (SAM septal distance $<10 \mathrm{~mm}$ ) or mild (distance $>10 \mathrm{~mm}$ ), as shown in Figure 1. Severe SAM, i.e. prolonged SAM septal contact for more than $30 \%$ of echocardiographic systole, occurred at rest in all 27 patients with haemodynamically proven resting obstruction, but in no patient with latent or no obstruction. Patients with latent obstruction usually had

The Fellowship of Postgraduate Medicine, 1986 


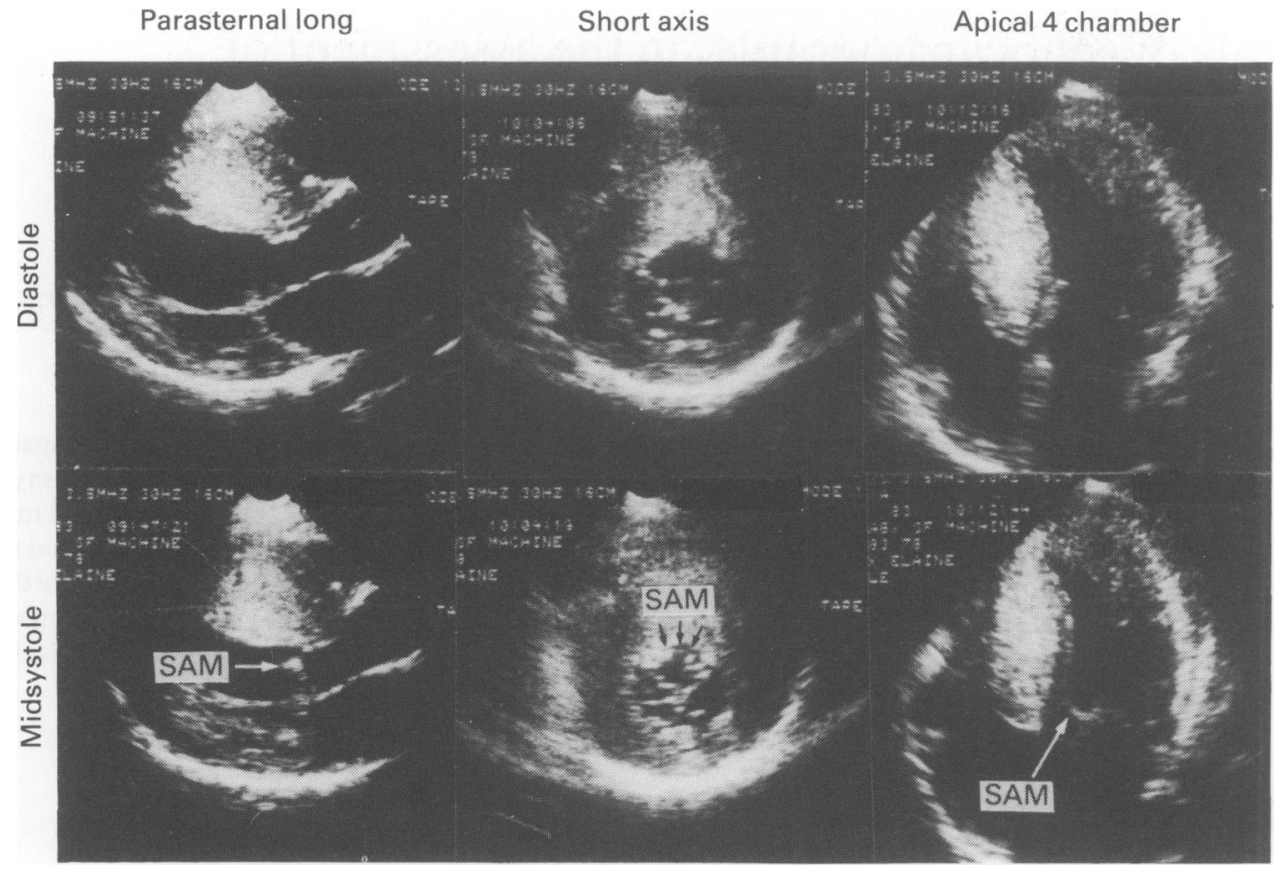

Figure 1 Diastolic and mid-systolic parasternal and apical 4 chamber frames from a patient with HCM, severe asymmetric hypertrophy and resting obstruction. The systolic anterior motion (SAM) of the mitral apparatus involves the chordae and anterior leaflet severely narrowing the LV outflow tract. SAM-septal contact is extensive involving much of the circumference (short axis arrows) of the LV outflow tract. Note that at the time of SAM-septal contact little LV area reduction has taken place in keeping with a large percentage of LV stroke volume yet to be ejected.

after the inhalation of amyl nitrite. Patients without obstruction usually did not have SAM (13/15) and provocation failed to induce moderate or severe SAM. Pollick et al. (1982) demonstrated the temporal relationship between SAM and the pressure gradient in 18 patients with resting obstruction (gradient $73 \pm 18 \mathrm{mmHg}$ ). The onset of SAM was an early systolic event occurring after just $6 \%$ of the systolic ejection period. The onset of the pressure gradient and SAM septal contract were almost simultaneous, occurring at $23 \pm 5 \%$ and $25 \pm 7 \%$ of the systolic ejection period respectively.

Precise quantitation of the pressure gradient is possible from good quality $\mathrm{M}$-mode studies. Henry et al. (1973) pioneered such attempts by measuring the LVOT area during systole, although this method was laborious and only accurate for larger pressure gradients. Pollick et al. (1984) developed a simple accurate method of quantitating a pressure gradient $>25 \mathrm{mmHg}$ based on the timing and duration of SAM septal contact.

We believe that 2D-echo studies show the chordae tendinae \pm anterior mitral leaflet tip to be responsible for lesser degrees of SAM. With severe SAM the body of the anterior mitral leaflet, and occasionally the posterior leaflet, is usually involved, and short axis views confirm significant narrowing of the LVOT over most of its circumference (Figure 1).

Doppler studies have further enhanced our ability to directly quantitate the degree of LVOT obstruction. In patients with obstruction, peak LVOT ejection velocities can be measured using either high pulse repetition frequency or continuous wave (CW) Doppler. With CW studies, care must be taken to distinguish the LVOT jet from that of associated mitral regurgitation (Hatle \& Angelsen, 1985) since they have similar direction and velocity. The pressure gradient (PG) is calculated from the modified Bernoulli equation being equal to 4 times the peak velocity $(\mathrm{V})^{2}$ $\left(\mathrm{PG}=4 \mathrm{~V}^{2}\right)$.

Doppler studies have also confirmed earlier angiographic and indicator dilution studies (Wigle $e t$ al., 1985) demonstrating a relationship between the severity of LVOT obstruction, the degree of SAM, and the severity of mitral regurgitation. Presumably this is due to increased distortion and lack of coaptation of the mitral leaflets with greater degrees of SAM. 


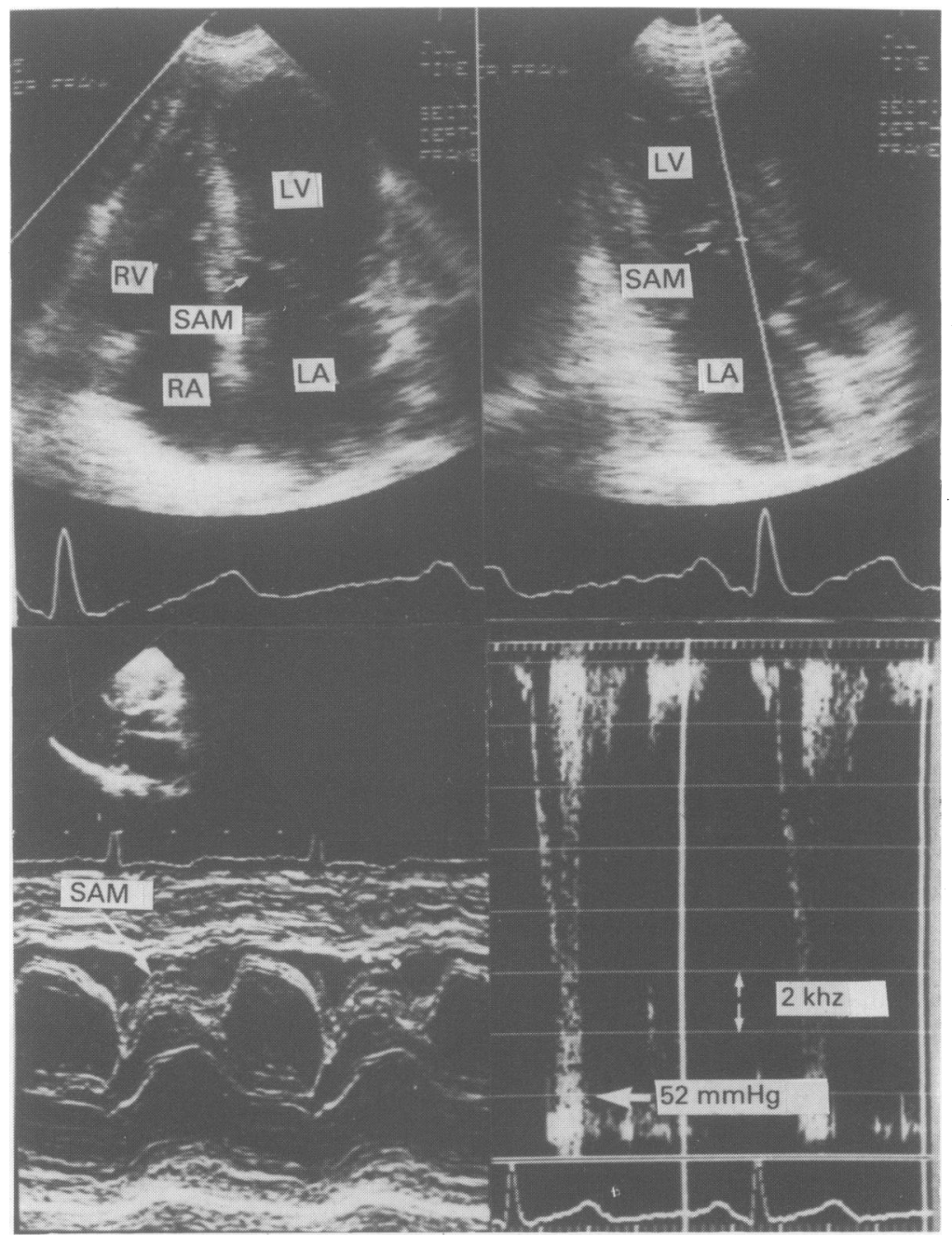

Figure 2 M-mode and Doppler assessment of the pressure gradient in obstructive HCM. The upper panels demonstrate severe SAM by 2D-echocardiography in the apical 4 chamber (left) and apical long axis (right) views. The arrow on the right upper panel indicates the Doppler sample volume just superior to the site of SAM - septal contact. A high velocity $\mathrm{LV}$ outflow jet (lower right) corresponding to a $52 \mathrm{mmHg}$ gradient is detected at this site. SAM - septal contact is also shown (lower left). $R V=$ right ventricle; $L V=$ left ventricle; $R A=$ right atrium; $\mathbf{L A}=$ left atrium.

The importance of the degree of asymmetric hypertrophy

Variations in the degree of asymmetric hypertrophy in patients with HCM have been recognized since Teare's (1958) original pathological studies. Two-dimensional echo studies from our laboratory (Wigle et al., 1985) as well as by Maron et al. (1981) and Shapiro \& McKenna (1983) have demonstrated significant anatomical variations in the degree of hypertrophy.
The hypertrophy is usually maximal in the proximal septum extending down its length to a variable degree. In adult patients with HCM proximal septal thickness is generally greater than $15 \mathrm{~mm}$ and occasionally exceeds $35 \mathrm{~mm}$. Circumferential extent of the hypertrophy medially and laterally from the septum is also variable. Occasionally apical or midventricular hyper- 
Table I Extent of asymmetric hypertrophy related to haemodynamic subgroups in hypertrophic cardiomyopathy

\begin{tabular}{lccccccc}
\hline Haemodynamic subgroup & No. of & \multicolumn{3}{c}{ Extent of septal hypertrophy } & $\begin{array}{c}\text { Anterolateral } \\
\text { extension }\end{array}$ & $\begin{array}{c}\text { Mean echo } \\
\text { point score }\end{array}$ \\
& cases & IVS $\mathrm{mm}$ & Basal 1/3 & Basal 2/3 & Whole septum & & \\
\hline Resting obstruction & 39 & $2.45 \pm 0.55$ & $8 \%$ & $20 \%$ & $72 \%^{*}$ & $83 \%$ & 8.57 \\
Latent obstruction & 34 & $1.89 \pm 0.35$ & $53 \%^{*}$ & $35 \%$ & $12 \%^{*}$ & $13 \%^{*}$ & $2.88^{*}$ \\
No obstruction & 27 & $2.09 \pm 0.57$ & $14 \%^{* *}$ & $26 \%$ & $59 \%^{* *}$ & $63 \%^{* *}$ & $6.04^{* *}$ \\
\hline
\end{tabular}

* $=\mathrm{P}<0.001$ latent $v s$ both resting and no obstruction

${ }^{* *}=\mathrm{P}<0.05$ no obstruction $v s$ resting obstruction

trophy predominates. In patients with resting obstruction the hypertrophied septum causes greater narrowing of the LVOT and often there is posterior wall hypertrophy.

To better define variations in the degree of asymmetric hypertrophy we studied 100 patients with good quality 2D-echocardiograms who had undergone haemodynamic classification. The degree of asymmetric hypertrophy was semiquantitated using a 2Decho point score with a maximum of 10 points given. One to 4 points was given to septal thickness measured at the leaflet tips in the "parasternal long axis view $(15-19 \mathrm{~mm}=1$ point, $20-24 \mathrm{~mm}=2$ points, $25-29 \mathrm{~mm}=3$ points, $>30 \mathrm{~mm}=4$ points). Up to 4 points were given for the length of asymmetric septal hypertrophy as determined from long axis and 4 chamber views (extension to papillary muscles -2 points, extension to apex -4 points). An additional 2 points were given for extension of hypertrophy to the anterolateral wall. Table I shows the differences in the extent of asymmetric hypertrophy in the different haemodynamic subgroups of HCM. Patients with latent obstruction had a less severe form of hypertrophy. In $53 \%$, the hypertrophy was localized to the proximal portion of the septum and anterolateral extension was uncommon (13\%). This is in keeping with our previous observations that these patients have less severe symptoms and rarely die of ventricular arrhythmias. Patients with resting obstruction had the greatest degree of hypertrophy with involvement of at least $2 / 3$ of the septum in $92 \%$ and very frequent anterolateral extension of the hypertrophy $(83 \%)$. Patients without obstruction had an intermediate degree of asymmetric hypertrophy with some having mild and others more severe hypertrophy. Patients with greater degrees of hypertrophy as shown by an echo point score of $>5$ had significantly higher degrees of NYHC 3-4 dyspnoea and angina, septal perforator compression, elevated LVEDP, and ventricular tachycardia (Wigle et al., 1985). Thus the degree of asymmetric hypertrophy is important to determine since it can be related to haemodynamic subgroup, symptoms, occurrence of septal perforator compression, diastolic relaxation and compliance, lifethreatening ventricular arrhythmias and thus prognosis.

Table II Echo-Doppler assessment of effects of therapy

1. Decreased or abolished obstruction

(a) decrease or abolition of SAM

(b) decreased LVOT velocity

(c) disappearance of systolic aortic notching

(d) normalized aortic systolic flow pattern

(e) decrease or abolition of mitral regurgitation

2. Improved diastolic function

(a) more rapid time to peak filling

(b) longer diastasis

(c) increased LV filling during early diastole

(d) lower atrial LV filling velocity

\section{Assessing the effects of therapy}

Therapeutic decisions are in large part determined by symptoms and haemodynamic subgroup (Wigle et al., 1985). Disopyramide and verapamil can reduce the degree of LVOT obstruction although myectomy is necessary in patients who are not adequately improved. Diastolic dysfunction may be improved by verapamil or nifedipine, the latter drug reserved for patients without obstruction. Improvement in or abolition of the LVOT obstruction and improved diastolic LV filling can be assessed by echo Doppler studies (Shapira et al., 1978; Wigle et al., 1985) as listed in Table II and shown in Figure 3.

\section{Acknowledgements}

The authors are grateful to Ms. Amanda Clark for her help in the preparation of this manuscript. This work was supported in part by the Canadian and Ontario Heart Foundation. 


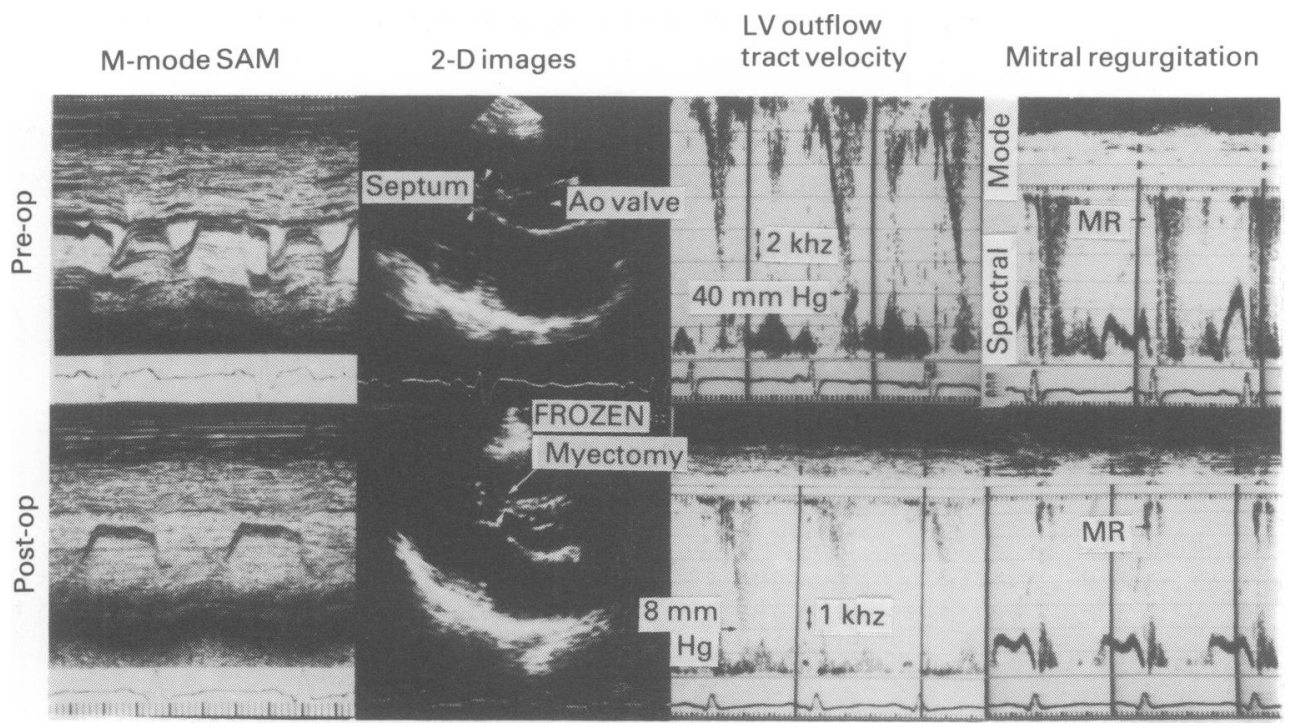

Figure 3 Relief of LVOT obstruction following surgical myectomy. Post-operatively the basal septum is thinned resulting in enlargement of the LVOT, abolition of SAM, reduction in the Doppler detected LVOT gradient and near disappearance of mitral regurgitation (MR).

\section{References}

GILBERT, B.W., POLLICK, C., ADELMAN, A.G. \& WIGLE, E.D. (1980). Hypertrophic cardiomyopathy: The subclassification by $\mathbf{M}$-mode echocardiography. American Journal of Cardiology, 45, 861.

HATLE, L. \& ANGELSEN, B. (1985). In Doppler Ultrasound in Cardiology. p. 205. Lea and Febiger: Philadelphia.

HENRY, W.L., CLARKE, C.E., GLANCY, D.L., EPSTEIN, S.E. (1973). Echocardiographic measurement of the left ventricular outflow gradient in idiopathic hypertrophic subaortic stenosis. New England Journal of Medicine, 288, 989.

MARON, B.J., GOTTDIENER, J.S. \& EPSTEIN, S.E. (1981). Patterns and significance of distribution of left ventricular hypertrophy in hypertrophic cardiomyopathy. American Journal of Cardiology, 48, 418.

MARTIN, R.P., RAKOWSKI, H., FRENCH, J. \& POPP, R.L. (1979). Idiopathic hypertrophic subaortic stenosis viewed by wide-angle, phased-array echocardiography. Circulation, 59, 1206.

POLLICK, C., MORGAN, C.D., GILBERT, B.W., RAKOWSKI, H. \& WIGLE, E.D. (1982). Muscular subaortic stenosis: The temporal relationship between systolic anterior motion of the anterior mitral leaflet and the pressure gradient. Circulation, 66, 1087.

POLLICK, C., RAKOWSKI, H. \& WIGLE, E.D. (1984). Mus- cular subaortic stenosis: the quantitative relationship between systolic anterior motion and the pressure gradient. Circulation, 69, 43.

POPP, R.L. \& HARRISON, D.C. (1969). Ultrasound in the diagnosis and evaluation of therapy in idiopathic hypertrophic subaortic stenosis. Circulation, 40, 905.

SCHAPIRA, J.N., STEMPLE, O.R., MARTIN, R.P., RAKOWSKI, H., STINSON, E.B. \& POPP, R.L. (1978). Single and twodimensional echocardiographic visualization of the effects of septal myectomy in idiopathic hypertrophic subaortic stenosis. Circulation, 58, 851.

SHAH, P.M., GRAMIAK, R. \& KRAMER, D.H. (1969). Ultrasound localization of left ventricular outflow obstruction in hypertrophic obstructive cardiomyopathy. Circulation, 40, 3.

SHAPIRO, L.M. \& McKENNA, W.J. (1983). Distribution of left ventricular hypertrophy in hypertrophic cardiomyopathy. Journal of the American College of Cardiology, 2, 437.

TEARE, R.D. (1958). Asymmetrical hypertrophy of the heart in young adults. British Heart Journal, 20, 1.

WIGLE, E.D., SASSON, Z., HENDERSON, M.A., RUDDY, T.D., FULOP, J., RAKOWSKI, H. \& WILLIAMS, W.G. (1985). Hypertrophic cardiomyopathy: the importance of the site and extent of hypertrophy. A review. Progress in Cardiovascular Disease, 28, 1. 